\title{
POROSITY OF THE FREE BOUNDARY FOR $p$-PARABOLIC TYPE EQUATIONS IN NON-DIVERGENCE FORM
}

\author{
GLEYDSON C. RICARTE
}

\begin{abstract}
In this article we establish the exact growth of the solution to the singular quasilinear $p$-parabolic free boundary problem in non-divergence form near the free boundary from which follows its porosity.
\end{abstract}

Keywords: Normalized $p$-Laplacian, singularly perturbed problems, Lipschitz regularity, porosity of the free boundary.

AMS Subject Classifications MSC 2010: 35K55, 35D40, 35B65, 35R35.

\section{INTRODUCTION}

In this paper we prove an uniform gradient estimate for solutions to a one phase free boundary problem involving singular $p$-parabolic type equations in nondivergence form. This estimate then allows us to obtain porosity of the free boundary. More precisely, let $\Omega$ be an open bounded domain of $\mathbb{R}^{n}, n \geq 2, T>0$. We consider the problem: Find $0 \leq u \in C((0, T) \times \Omega)$ such that

$$
\left\{\begin{aligned}
\Delta_{p}^{N} u-u_{t} & =f \cdot \chi_{\{u>0\}} & & \text { in } \Omega_{T} \\
u & =\varphi & & \text { on } \partial_{p} \Omega_{T},
\end{aligned}\right.
$$

where $p \in(1,+\infty), \Delta_{p}^{N}$ is the normalized $p$-Laplacian defined by

$$
\begin{aligned}
\Delta_{p}^{N} u: & =\frac{1}{p}|\nabla u|^{2-p} \operatorname{div}\left(|\nabla u|^{p-2} \nabla u\right) \\
& =\frac{1}{p} \Delta u+\frac{p-2}{p} \Delta_{\infty}^{N} u,
\end{aligned}
$$

and $\Delta_{\infty}^{N} u:=\left\langle D^{2} u \frac{\nabla u}{|\nabla u|}, \frac{\nabla u}{|\nabla u|}\right\rangle$ is a normalized infinity Laplacian, $f: \Omega_{T} \rightarrow \mathbb{R}$ is a function non-increasing in $t$ and satisfying for two positive constants $c_{0}$ and $c_{1}$

$$
0<c_{0} \leq f(x, t) \leq c_{1}<\infty \quad \text { in } \quad \Omega_{T},
$$

$\varphi(x, 0)=0$ in $\Omega$ and $\varphi$ is non-decreasing in $t$. We are interested in regularity theory and geometric properties of $\partial\{u>0\}$. For this, we use the regularity theory of normalized $p$-Laplacian parabolic equations which was recently developed in 2. 11, 17. When we consider free boundary problems, optimal regularity results and sharp non-degeneracy are crucial for further analysis of the set $\partial\{u>0\}$. In this direction, just recently we have the work [16].

Equation (1.1) is the degenerate fully nonlinear version of the parabolic problem studied by H. Shahgholian \& G. Ricarte, J.M. Urbano and R. Teymurazyan in 18 and [16] respectively. They proved optimal regularity and non-degeneracy

estimates for the solution. As a consequence, they were able to obtain finite speed 
of propagation of the set $\partial\{u>0\}$ and also Hausdorff measure estimate of the free boundary $\partial\{u>0\}$ with respect to the parabolic metric.

In analogy with [16, what we want to prove is that, at each time level, the ndimensional Lebesgue measure of the free boundary is zero because it is porous. We prove this by obtaining a non-degeneracy result and by controlling the growth rate of the solution near the free boundary. As in [16, the solution to (1.1) is derived from an approximating family of functions, which are solutions to some Dirichlet problems. More precisely, we consider the following singular perturbation problem

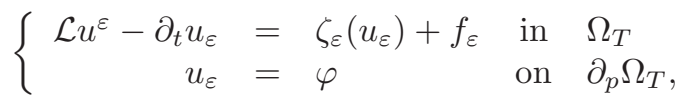

where $\mathcal{L} v:=\frac{1}{p} \Delta v+\frac{p-2}{p} \Delta_{\infty}^{N} v$. The singularly perturbed potential $\zeta_{\varepsilon}(\cdot)$ is a suitable approximation of a multiple of the Dirac mass $\delta_{0}$. Our objective is to study the limit problem as $\varepsilon \rightarrow 0$ and analyze the free boundary in the context of geometric measure theory. In the next section we give more details of our results and hypothesis.

Acknowledgments. GCR thanks the Analysis research group of UFC for fostering a pleasant and productive scientific atmosphere. The author research has been partially funded by FUNCAP-Brazil.

\section{MAIN RESUlts}

Let us now describe in more details our results and hypothesis.

2.1. Function spaces and notations. Let us fix the notation of geometric quantities that we are going to use in this work. Given a bounded domain $\Omega \subset \mathbb{R}^{n}$, with a smooth boundary $\partial \Omega$, we define, for $T>0, \Omega_{T}=\Omega \times(0, T]$, its lateral boundary $\Sigma=\partial \Omega \times(0, T)$ and its parabolic boundary $\partial_{p} \Omega_{T}=\Sigma \cup(\Omega \times\{0\})$. For $X_{0} \in \mathbb{R}^{n}$, $t_{0} \in \mathbb{R}$ and $\tau>0$, we denote

$$
\begin{aligned}
B_{\tau}\left(X_{0}\right) & :=\left\{x \in \mathbb{R}^{n}:\left|X-X_{0}\right|<\tau\right\}, \\
Q_{\tau}\left(X_{0}, t_{0}\right) & :=B_{\tau}\left(X_{0}\right) \times\left(t_{0}-\tau^{2}, t_{0}+\tau^{2}\right), \\
Q_{\tau}^{-}\left(X_{0}, t_{0}\right) & :=B_{\tau}\left(X_{0}\right) \times\left(t_{0}-\tau^{2}, t_{0}\right],
\end{aligned}
$$

and, for a set $K \subset \mathbb{R}^{n+1}$ and $\tau>0$,

$$
\mathcal{N}_{\tau}(K):=\bigcup_{\left(X_{0}, t_{0}\right) \in K} Q_{\tau}\left(X_{0}, t_{0}\right) \quad \text { and } \quad \mathcal{N}_{\tau}^{-}(K):=\bigcup_{\left(X_{0}, t_{0}\right) \in K} Q_{\tau}^{-}\left(X_{0}, t_{0}\right) .
$$

Let's start the section this section defining viscosity solutions of equation $\mathcal{L} v-$ $v_{t}=f(X, t)$ in $\Omega_{T}$ and fix the notation. For $p \in(1,+\infty)$, the operator

$$
\mathcal{L} v:=\frac{1}{p} \Delta v+\frac{p-2}{p} \Delta_{\infty}^{N} v
$$

is singular at $\{\nabla u=0\}$ and so the definition uses USC and LSC envelopes of the operator, see 9. Afterwards, we give some remarks to enlighten some basic features of solutions. To this end, given a function $h$ defined in a set $\mathcal{D}$, we need to introduce its upper semicontinuous envelope $h^{\star}$ and lower semicontinuous envelope 
$h_{\star}$ defined by

$$
\begin{aligned}
& h^{\star}(x, t):=\lim _{r \downarrow 0} \sup \left\{h(y, t):(y, t) \in \overline{Q_{r}(x, t)} \cap \mathcal{D}\right\} \\
& h_{\star}(x, t):=\liminf _{r \downarrow 0}\left\{h(y, t):(y, t) \in \overline{Q_{r}(x, t)} \cap \mathcal{D}\right\}
\end{aligned}
$$

as functions defined in $\overline{\mathcal{D}}$. The definition for viscosity solutions is the following.

Definition 2.1. A continuous function $v$ is a viscosity subsolution (supersolution) to the equation

$$
\mathcal{L} v-v_{t}=g(X, t) \quad \text { in } \quad \Omega_{T}
$$

if and only if for all $\left(X_{0}, t_{0}\right) \in \Omega_{T}$ and $\phi \in C^{2}\left(\Omega_{T}\right)$ such that $u-\phi$ attains a local maximum (minimum) at $\left(X_{0}, t_{0}\right)$, then

(1) $\mathcal{L} \phi\left(X_{0}, t_{0}\right)-\phi_{t}\left(X_{0}, t_{0}\right) \geq g\left(X_{0}, t_{0}\right) \quad($ resp. $\leq)$ if $\nabla \phi\left(X_{0}, t_{0}\right) \neq 0$

(2) $\Delta \phi\left(X_{0}, t_{0}\right)+(p-2) \lambda_{\max }\left(D^{2} \phi\left(X_{0}, t_{0}\right)\right)-\phi_{t}\left(X_{0}, t_{0}\right) \geq g\left(X_{0}, t_{0}\right) \quad($ resp. $\leq)$ if $\nabla \phi\left(X_{0}, t_{0}\right)=0$ and $p \geq 2$

(3) $\Delta \phi\left(X_{0}, t_{0}\right)+(p-2) \lambda_{\min }\left(D^{2} \phi\left(X_{0}, t_{0}\right)\right)-\phi_{t}\left(X_{0}, t_{0}\right) \geq g\left(X_{0}, t_{0}\right) \quad($ resp. $\leq)$ if $\nabla \phi\left(X_{0}, t_{0}\right)=0$ and $1<p<2$.

We say that $u$ is a viscosity solution of (2.2) in $\Omega_{T}$ if it is both a viscosity sub-and supersolution.

We will use the following two properties of the viscosity solutions of (2.2). The first one is the comparison principle, which can be found in Theorem 3.2 in [3].

Proposition 2.1. Let $u$ lower-semicontinuous and $v$ upper-semicontinuous. Suppose that $v$ is a subsolution, and $u$ a supersolution to (2.2) with $g \in C\left(\overline{Q_{1}}\right), g>0$ and $1 \leq p \leq \infty$. Further, suppose that $v \leq u$ on $\partial_{p} Q_{1}$. Then $v \leq u$ in $\overline{Q_{1}}$.

The second one is the stability of viscosity solutions of (2.2).

Proposition 2.2 (Stability). Let $\left\{u_{k}\right\}$ be a sequence of viscosity solution of (2.2) in $Q_{1}, u_{k}$ converge locally uniformly to $u$ and $g_{k} \rightarrow g$ locally uniformly in $Q_{1}$. Then $u$ is a viscosity solution of (2.2) in $Q_{1}$.

We need to clarify what is a Lipschitz function defined in a space-time domain.

Definition 2.2. Let $\mathcal{D} \subset \mathbb{R}^{n} \times \mathbb{R}$. We say that $v \in$ Lip $_{\text {loc }}(1,1 / 2)(\mathcal{D})$ if, for every compact $K \Subset \mathcal{D}$, there exists a constant $C=C(K)$ such that

$$
|v(x, t)-v(y, s)| \leq C\left(|x-y|+|t-s|^{\frac{1}{2}}\right),
$$

for every $(x, t),(y, s) \in K$. If the constant $C$ does not depend on the set $K$ we say $v \in \operatorname{Lip}(1,1 / 2)(\mathcal{D})$.

We also define the $\operatorname{Lip}(1,1 / 2)(\mathcal{D})$ seminorm in $\mathcal{D}$

$$
[v]_{\operatorname{Lip}(1,1 / 2)(\mathcal{D})}:=\sup _{(x, t),(y, s) \in \mathcal{D}} \frac{|v(x, t)-v(y, s)|}{|x-y|+|t-s|^{1 / 2}}
$$

and the $\operatorname{Lip}(1,1 / 2)(\mathcal{D})$ norm in $\mathcal{D}$

$$
\|v\|_{\operatorname{Lip}(1,1 / 2)(\mathcal{D})}:=\|v\|_{L^{\infty}(\mathcal{D})}+[v]_{\operatorname{Lip}(1,1 / 2)(\mathcal{D})} .
$$


2.2. Hypothesis. Throughout the paper, for the study of problem $E_{\varepsilon}$

- $f_{\varepsilon}(x, t) \in C^{1, \alpha}\left(\overline{\Omega_{T}}\right)$, is non-increasing in $t$ and satisfies

$$
0<c_{0} \leq f_{\varepsilon}(x, t) \leq c_{1}<\infty \quad \text { in } \quad \Omega_{T}
$$

and

$$
\left\|\nabla f_{\varepsilon}\right\|_{\infty} \leq C .
$$

- The Dirichlet data $0 \leq \varphi(x, t) \in C^{1, \alpha}\left(\partial_{p} \Omega_{T}\right)$, is non-decreasing in $t$ and satisfies $\varphi(x, 0)=0$.

These are the same hypothesis assumed in [16].

2.3. Existence and optimal regularity. Our existence theorem relies on a singularly perturbed analysis. To this end, we shall define the perturbed term $\zeta_{\varepsilon}$ : $\mathbb{R}_{+} \rightarrow \mathbb{R}_{+}$satisfying

$$
0 \leq \zeta_{\varepsilon}(s) \leq \frac{1}{\varepsilon} \chi_{(0, \varepsilon)}(s), \quad \forall s \in \mathbb{R}_{+}
$$

and the corresponding problem $E_{\varepsilon}$. For example, it can be built as an approximation of unity

$$
\zeta_{\varepsilon}(s):=\frac{1}{\varepsilon} \zeta\left(\frac{s}{\varepsilon}\right),
$$

where $\zeta$ is a nonnegative smooth real function with supp $\zeta=[0,1]$, such that

$$
\|\zeta\|_{\infty} \leq 1 \text { and } \int_{\mathbb{R}} \zeta(s) d s<\infty .
$$

This existence result is based on Perron's method. We state before following theorem independently of the $E_{\varepsilon}$ context, since it may be of independent interest.

Proposition 2.3 (Perron's Method). Let $g:[0, \infty) \rightarrow \mathbb{R}$ be a bounded and Lipschitz function and $\mathcal{F}: \Omega_{T} \times \mathbb{R}^{n} \times S y m(n) \rightarrow \mathbb{R}$ a degenerate elliptic operator satisfying the following monotonicity condition

$$
\mathcal{F}(x, t, \vec{p}, N) \leq \mathcal{F}(x, t, \vec{p}, M) \quad \text { whenever } \quad N \leq M,
$$

for any $\vec{p} \in \mathbb{R}^{n}$ and $N, M \in \operatorname{Sym}(n)$. If the equation

$$
u_{t}-\mathcal{F}\left(x, t, \nabla u, D^{2} u\right)=g(u)+f(X, t)
$$

with $f \in C\left(\Omega_{T}\right)$, admits subsolution and supersolution $\underline{u}, \bar{u} \in C^{0}\left(\overline{\Omega_{T}}\right)$ respectively, and $\underline{u}=\bar{u}=\varphi \in W^{2, \infty}\left(\partial_{p} \Omega_{T}\right)$, then given the set of functions

$$
\mathcal{S}:=\left\{w \in C\left(\overline{\Omega_{T}}\right) \mid w \text { is a supersolution to (2.6), and } \underline{u} \leq w \leq \bar{u}\right\},
$$

the function

$$
v(x, t):=\inf _{w \in \mathcal{S}} w(x, t)
$$

is a continuous viscosity solution to (2.6), safisfying $u=\varphi$ in $\partial_{p} \Omega_{T}$.

Proof. Using Proposition 2.1, the proof follows exactly as the one of 16, Theorem $3.1]$. 
In order to prove the existence result of the problem $\left[E_{\varepsilon}\right.$, we choose to approximate the equation $E_{\varepsilon}$ with a regularized problem. For $\delta>0$, let $u_{\varepsilon, \delta}$ be smooth and satisfying that

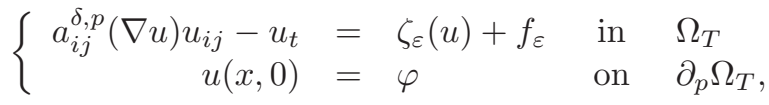

where

$$
a_{i j}^{\delta, p}(\vec{\eta}):=\frac{1}{p} \delta_{i j}+\frac{p-2}{p} \frac{\eta_{i} \eta_{j}}{|\vec{\eta}|^{2}+\delta} .
$$

Note that, $\left(a_{i j}^{\delta, p}\right)_{i, j}$ is uniformly parabolic with

$$
\lambda|\xi|^{2} \leq a_{i j}^{\delta, p}(\vec{\eta}) \xi_{i} \xi_{j} \leq \Lambda|\xi|^{2},
$$

where $\Lambda=\max \left(\frac{p-1}{p}, \frac{1}{p}\right)$ and $\lambda=\min \left(\frac{p-1}{p}, \frac{1}{p}\right)$. Since $p>1$, it follows from classical quasilinear equation theory (see e.g. [12, Theorem 4.4]) and the Schauder estimates that, for each $\delta>0$, there exists a unique solution $u_{\varepsilon, \delta} \in C^{3, \beta}\left(\Omega_{T}\right) \cap$ $C\left(\overline{\Omega_{T}}\right)$ of (2.8) with $p>1$.

Theorem 2.1. Let $u_{\varepsilon, \delta}: \bar{\Omega} \times(0, T) \rightarrow \mathbb{R}$ be a viscosity solution of the regularized problem (2.8). Then there exists a viscosity subsolution $u^{\star}: \bar{\Omega} \times(0, T) \rightarrow \mathbb{R}$ and a viscosity supersolution $u_{\star}: \bar{\Omega} \times(0, T) \rightarrow \mathbb{R}$ of the original problem $\left[E_{\varepsilon}\right]$, which have the form

$$
\begin{aligned}
u_{\varepsilon}^{\star}(x, t) & :=\lim _{\delta \rightarrow 0} \inf \left\{u_{\varepsilon, \mu}(\xi): \xi \in B_{\delta}(x, t), 0<\mu<\delta\right\} \\
u_{\star, \varepsilon}(x, t) & :=\lim _{\delta \rightarrow 0} \sup \left\{u_{\varepsilon, \mu}(\xi): \xi \in B_{\delta}(x, t), 0<\mu<\delta\right\}
\end{aligned}
$$

In particular, the problem (E) has a viscosity solution $\left\{u_{\varepsilon}\right\}$. Moreover, there exists a constant $\Upsilon=\Upsilon\left(n, \lambda, \Lambda, c_{0}\right)$, such that

$$
0 \leq u_{\varepsilon} \leq \Upsilon \text {. }
$$

Proof. For a proof of first part of the Theorem, we refer the reader to 11. The existence of viscosity solution to $E_{\varepsilon}$ follows from Proposition 2.3. To prove (2.10), let $v^{\varepsilon}:=u_{\varepsilon}-\|\varphi\|_{\infty}$. Note that $v^{\varepsilon} \leq 0$ on $\partial_{p} \Omega_{T}$ and

$$
a_{i j}^{\delta, p}\left(\nabla v^{\varepsilon}\right) v_{i j}^{\varepsilon}-\partial_{t} v^{\varepsilon}=a_{i j}^{\delta, p}\left(\nabla u_{\varepsilon}\right) D_{i j} u_{\varepsilon}-\partial_{t} u_{\varepsilon} \geq c_{0} .
$$

This means that $v_{\varepsilon} \in \underline{\mathcal{S}}\left(\frac{\lambda}{n}, \Lambda, c_{0}\right)$. The ABP estimate ([19, Theorem 3.14]) then implies

$$
\sup _{\Omega_{T}}\left(v_{\varepsilon}\right)^{+} \leq C\left(\lambda, \Lambda, n, c_{0}\right) .
$$

Thus, $u_{\varepsilon} \leq\|\varphi\|_{\infty}+C\left(\lambda, \Lambda, n, c_{0}\right)=: \Upsilon$.

In order to prove the nonnegativity of $u_{\varepsilon}$ we assume the contrary, i.e. that $A_{\varepsilon}:=\left\{(x, t) \in \Omega_{T}: u_{\varepsilon}(x, t)<0\right\} \neq \emptyset$. Since $\zeta_{\varepsilon}$ is supported in $[0, \varepsilon]$, then

$$
a_{i j}^{\delta, p}\left(\nabla u_{\varepsilon}\right) D_{i j} u_{\varepsilon}-\partial_{t} u_{\varepsilon}=f_{\varepsilon} \leq c_{1} \text { in } A_{\varepsilon},
$$

which means that $u_{\varepsilon} \in \overline{\mathcal{S}}\left(\frac{\lambda}{n}, \Lambda, c_{1}\right)$. Another application of the ABP estimate provides that $u_{\varepsilon} \geq 0$ in $A_{\varepsilon}$, which is a contradiction.

In order to pass to the limit as $\varepsilon \rightarrow 0^{+}$to obtain a solution of (1.1) we need sharp estimates uniform in $\varepsilon \in(0,1]$. Our main contribution is the following. 
Theorem 2.2. Let $u_{\varepsilon}$ be a viscosity solution of $E_{\varepsilon}$. Let $K \subset \Omega_{T}$ be compact and $\tau>0$ be such that $\mathcal{N}_{2 \tau}(K) \subset \Omega_{T}$. Then there exists a constant $L=$ $L\left(\tau, \lambda, \Lambda, c_{0}, c_{1},\|\nabla f\|_{\infty},\|\varphi\|_{\infty}, K\right)$ (in particular, not depending on $\varepsilon$ ) such that

$$
\left\|u_{\varepsilon}\right\|_{L i p(1,1 / 2)} \leq L .
$$

Theorem 2.2 gives us the necessary compactness to pass to the limit as $\varepsilon \rightarrow$ $0^{+}$obtaining a viscosity solution of (1.1). As a consequence, we also have sharp regularity.

Theorem 2.3. Let $u$ be a viscosity solution of (1.1). Let $K \subset \Omega_{T}$ be compact and $\tau>0$ be such that $\mathcal{N}_{2 \tau}(K) \subset \Omega_{T}$. Then there exists a constant $L=$ $L\left(\tau, \lambda, \Lambda, c_{0}, c_{1},\|\nabla f\|_{\infty},\|\varphi\|_{\infty}, K\right)$ such that

$$
\|u\|_{L i p(1,1 / 2)(K)} \leq L .
$$

This regularity result and some other properties of the free boundary problem (1.1) are proved in Section 3 .

2.4. Some properties of the free boundary. In this section we establish the exact growth of the solution near the free boundary, from which we deduce the porosity of its time level sets.

Definition 2.3. A set $E \subset \mathbb{R}^{n}$ is called porous with porosity $\delta>0$, if there exists $R>0$ such that

$$
\forall x \in E, \quad \forall r \in(0, R), \quad \exists y \in \mathbb{R}^{n} \text { such that } B_{\delta r}(y) \subset B_{r}(x) \backslash E .
$$

A porous set of porosity $\delta$ has Hausdorff dimension not exceeding $n-c \delta^{n}$, where $c=c(n)>0$ is a constant depending only on $n$. In particular, a porous set has Lebesgue measure zero.

The main result of this section is as follows.

Theorem 2.4. Let $u$ be a solution of (1.1). Then, for every compact set $K \subset \Omega_{T}$ and every $t_{0} \in(0, T)$, the set

$$
\partial\{u>0\} \cap K \cap\left\{t=t_{0}\right\}
$$

is porous in $\mathbb{R}^{n}$, with porosity depending only on $\Upsilon$ and $\operatorname{dist}\left(K, \partial_{p} \Omega_{T}\right)$. In other words,

$$
\mathcal{L}^{n}\left(\partial\{u>0\} \cap K \cap\left\{t=t_{0}\right\}\right)=0 .
$$

The proof is based on the exact growth of the solution of the problem (1.1) near the free boundary. This result is proved in Section 4

Theorem 2.5. Assume $p \in(1,+\infty)$ and let $u$ be a viscosity solution of the problem (1.1). Then there exist two positive constants $d_{0}=d_{0}\left(n, p, c_{0}\right)$ and $D_{0}=$ $D_{0}\left(n, p, c_{0}, c_{1}\right)$ such that for every compact set $K \Subset \Omega_{T},\left(X_{0}, t_{0}\right) \in(\partial\{u>0\}) \cap K$, the following estimates hold

$$
d_{0} r^{2} \leq \sup _{B_{r}\left(X_{0}\right)} u\left(\cdot, t_{0}\right) \leq D_{0} r^{2} .
$$




\section{UNIFORM EstimAtes IN TIME AND SPACE FOR THE PERTURBED PROBLEM}

This section discusses regularity for the viscosity solution $u_{\varepsilon}$ of the singular perturbation problem $E_{\varepsilon}$ for $p \in(1,+\infty)$. First of all we show Lipschitz continuity of the viscosity solution $u_{\varepsilon}$ with respect to $x$ using a Bernstein type argument. The strategy to show Lipschitz regularity is based on the works [11, 16] but it turns out that the result is not true for $p=1$ since the constant $\bar{L}$ (see Proposition 3.1) blows up for $p \rightarrow 1$. Finally, we will show that bound on the gradients implies limitation in the seminorm $\operatorname{Lip}(1,1 / 2)$.

3.1. Uniform spatial regularity. In order to prove Theorem 2.2 we choose a regularized problem given by (2.8). We start with the uniform Lipschitz regularity in the spatial variables.

Proposition 3.1. If $\left\{u_{\varepsilon, \delta}\right\}$ is a family of solutions of (2.8), and (2.3)-(2.4) hold, then there exists a constant $\bar{L}=\bar{L}\left(\tau, \lambda, \Lambda, c_{0}, c_{1},\|\nabla f\|_{\infty},\|\varphi\|_{\infty}, K\right)>0$, independent of $\delta>0$ and $\varepsilon \in(0,1)$, such that

$$
\left|\nabla u_{\varepsilon, \delta}(x, t)\right| \leq \bar{L}\left(1+\frac{1}{\operatorname{dist}\left((x, t), \partial_{p} \Omega_{T}\right)^{2}}\right), \forall(x, t) \in \Omega_{T} .
$$

Proof. Fix $\varepsilon>0$ and for each $\delta>0$, let $u_{\varepsilon, \delta}=u^{\delta}$ (for simplicity) be a smooth viscosity solution to (2.8). To show (3.1), initially, since $\zeta_{\varepsilon}=0$ in $\left\{u^{\delta}>\varepsilon\right\}$, we conclude from up to the boundary parabolic regularity theory (see [19, Theorem 4.19] and [20, Theorem 2.5]) that

$$
\left|\nabla u^{\delta}\right| \leq C\left(\left\|u^{\delta}\right\|_{\infty}+\left\|f_{\varepsilon}\right\|_{n+1}+\|\varphi\|_{\infty}\right)
$$

in this region, where $C$ does not depend on $\varepsilon$ and $\delta>0$. The result then follows from (2.4) and (2.10), passing to the limit $\delta \rightarrow 0$. To show a Lipschitz estimate with respect to $x$ in $\left\{u^{\delta} \leq \epsilon\right\}$, we define $v(x, t)=\left(\left|D u^{\delta}\right|^{2}+\delta\right)^{1 / 2}$ and consider the function

$$
w(x, t)=\xi(x, t) \cdot v(x, t)+\mu u^{\delta}(x, t)^{2},
$$

where $\mu=\frac{\Gamma}{2 \varepsilon^{2}}$, for some $\Gamma \geq 0,0 \leq \xi \leq 1$ is a positive smooth function that vanishes on the parabolic boundary $\partial_{p} \Omega_{T}$. Let $\left(x_{0}, t_{0}\right) \in\left\{u^{\delta} \leq \varepsilon\right\}$ be a point where $w$ takes its maximum in $\Omega_{T} \cup \partial_{p} \Omega_{T}$. We can assume without loss of generality that

$$
\left|\nabla u^{\delta}\left(x_{0}, t_{0}\right)\right| \geq 1 \text {. }
$$

In fact, if $\left|\nabla u^{\delta}\right|<1$, then if $0<\delta \leq 1 / 2$,

$$
\begin{aligned}
\left|\nabla u^{\delta}(x, t)\right| & \leq v(x, t) \leq w(x, t) \leq w\left(x_{0}, t_{0}\right) \\
& =\xi\left(x_{0}, t_{0}\right) v\left(x_{0}, t_{0}\right)+\mu u^{\delta}\left(x_{0}, t_{0}\right)^{2} \\
& \leq\|\xi\|_{\infty} \sqrt{1+\delta}+\varepsilon^{2} \mu \leq \sqrt{1+\delta}+\Gamma \leq 2+\Gamma:=\bar{L} .
\end{aligned}
$$

First we suppose that $\left(x_{0}, t_{0}\right) \notin \partial_{p} \Omega_{T}$. At that point $D^{2} w\left(x_{0}, t_{0}\right)$ is negative definite. We define the coeficient matrix $a_{i j}^{p, \delta}$ as in (2.9). Since the matrix $\left(a_{i j}^{p, \delta}\left(D u^{\delta}(x, t)\right)\right)_{i j}$ is negative semi-definite for all points $(x, t)$ we have

$$
\sum_{i, j} a_{i j}^{\delta, p}\left(\nabla u^{\delta}\left(x_{0}, t_{0}\right)\right) w_{i j}\left(x_{0}, t_{0}\right) \leq 0
$$


Then,

$$
\begin{aligned}
0 & \leq w_{t}\left(x_{0}, t_{0}\right)-\sum_{i, j} a_{i j}^{\delta, p}\left(\nabla u^{\delta}\left(x_{0}, t_{0}\right)\right) w_{i j}\left(x_{0}, t_{0}\right) \\
& =\xi\left(v_{t}-\sum_{i, j} a_{i j}^{p, \delta}\left(D u^{\delta}\right) v_{i j}\right)+v\left(\xi_{t}-\sum_{i, j} a_{i j}^{p, \delta}\left(D u^{\delta}\right) \xi_{i j}\right) \\
& +2 \lambda u^{\delta}\left(u_{t}^{\delta}-\sum_{i, j} a_{i j}^{p, \delta}\left(D u^{\delta}\right) u_{i j}^{\delta}\right)-2 \sum_{i, j} a_{i j}^{p, \delta}\left(D u^{\delta}\right) \xi_{j} v_{i} \\
& -2 \lambda \sum_{i, j} a_{i j}^{p, \delta}\left(D u^{\delta}\right) u_{i}^{\delta} u_{j}^{\delta}
\end{aligned}
$$

holds at $\left(x_{0}, t_{0}\right)$. We estimate each term separately to obtain the desired inequality. The third term on the right hand side is

$$
2 \mu u^{\delta}\left(u_{t}^{\delta}-\sum_{i, j} a_{i j}^{p, \delta}\left(D u^{\delta}\right) u_{i j}^{\delta}\right)=-2 \mu u^{\delta}\left(f_{\epsilon}+\zeta_{\epsilon}\left(u^{\delta}\right)\right)
$$

because $u^{\delta}$ is a classical solution of the approximating problem. Now, we consider

$$
\xi\left(v_{t}-\sum_{i, j} a_{i j}^{p, \delta}\left(D u^{\delta}\right) v_{i j}\right) .
$$

We differentiate the equation (3.4) with respect to $x_{k}$, multiply $u_{t k}^{\delta}$ with $\frac{u_{k}^{\delta}}{v}$, and sum from $1, \ldots, n$ to obtain

$$
\begin{aligned}
v_{t} & =\frac{1}{p} \frac{1}{v} \sum_{i, k} u_{k}^{\delta} u_{i i k}^{\delta}+\frac{p-2}{p} \frac{1}{v^{3}} \sum_{i, j, k} u_{i}^{\delta} u_{j}^{\delta} u_{k}^{\delta} u_{i j k}^{\delta} \\
& +\frac{p-2}{p} \frac{2}{v^{3}} \sum_{i, j, k} u_{i}^{\delta} u_{i j}^{\delta} u_{k}^{\delta} u_{j k}^{\delta}-\frac{p-2}{p} \frac{2}{v^{5}}\left(\sum_{i, j} u_{i}^{\delta} u_{j}^{\delta} u_{i j}^{\delta}\right)^{2} \\
& -\frac{1}{v} \sum_{k}\left\{D_{k} f_{\epsilon} u_{k}^{\delta}+\epsilon^{-2} \zeta^{\prime}\left(u_{k}^{\delta}\right)^{2}\right\}
\end{aligned}
$$

The second derivatives of $v$ are

$$
v_{i j}=\frac{1}{v} \sum_{k} u_{i k}^{\delta} u_{j k}^{\delta}+\frac{1}{v} \sum_{k} u_{k}^{\delta} u_{i j k}^{\delta}-\frac{1}{v^{3}} \sum_{k}\left(u_{k}^{\delta} u_{i k}^{\delta}\right) \sum_{\ell}\left(u_{\ell}^{\delta} u_{j \ell}^{\delta}\right),
$$

and thus we have by a straightforward calculation

$$
\begin{aligned}
v_{t}-\sum_{i, j} a_{i j}^{p, \delta}\left(D u^{\delta}\right) v_{i j} & \leq-\frac{1}{p} \frac{1}{v} \sum_{i, k}\left(u_{i k}^{\delta}\right)^{2}-\frac{p-2}{p} \frac{1}{v^{5}}\left(\sum_{i, j} u_{i}^{\delta} u_{j}^{\delta} u_{i j}^{\delta}\right)^{2} \\
& +\frac{p-1}{p} \Lambda \frac{1}{v^{3}}|D v|^{2} v^{2}-\frac{1}{v} \sum_{k} D_{k} f_{\epsilon} u_{k}^{\delta}-\frac{1}{v} \epsilon^{-2} \zeta^{\prime}\left|D u^{\delta}\right|^{2}
\end{aligned}
$$


Note that

$$
-\frac{1}{p} \frac{1}{v} \sum_{i, k}\left(u_{i k}^{\delta}\right)^{2}-\frac{p-2}{p} \frac{1}{v^{5}}\left(\sum_{i, j} u_{i}^{\delta} u_{j}^{\delta} u_{i j}^{\delta}\right)^{2} \leq 0
$$

since

$$
\frac{1}{p} \sum_{i, k}\left(u_{i k}^{\delta}\right)^{2}+\frac{p-2}{p}\left(\sum_{i, j} u_{i j}^{\delta} \frac{u_{i}^{\delta}}{v} \frac{u_{j}^{\delta}}{v}\right)^{2} \geq \lambda \sum_{i, k}\left(u_{i k}^{\delta}\right)^{2} \geq 0
$$

and we have

$$
v_{t}-\sum_{i, j} a_{i j}^{p, \delta}\left(D u^{\delta}\right) v_{i j} \leq \frac{1}{v}\left(\Lambda|D v|^{2}+v \cdot\left\|D f_{\epsilon}\right\|_{\infty}+\epsilon^{-2} \zeta^{\prime}\left|D u^{\delta}\right|^{2}\right)
$$

In order to estimate the fourth term in (3.3) we use the fact that $w_{i}=\xi_{i} v+$ $\xi v_{i}+2 \lambda u^{\delta} u_{i}^{\delta}=0$ in $\left(x_{0}, t_{0}\right)$ and get

$$
\begin{aligned}
\xi \frac{|D v|^{2}}{v} & \leq \frac{v}{\xi}\left(|D \xi|^{2}+4 \mu\left|u^{\delta}\right||D \xi|+4\left(\mu u^{\delta}\right)^{2}\right) \\
& \leq \frac{5 v}{\xi}\left(|D \xi|^{2}+\left(\mu u^{\delta}\right)^{2}\right) .
\end{aligned}
$$

Hence

$$
\begin{aligned}
-2 \sum_{i, j} a_{i j}^{p, \delta}\left(D u^{\delta}\right) \xi_{j} v_{i} & \leq \frac{2 v}{\xi} \frac{1+|p-2|}{p}|D \xi|^{2}+\frac{4 v}{\xi} \frac{1+|p-2|}{p} \mu u^{\delta}|D \xi| \\
& \leq 4 \frac{1+|p-2|}{p} \frac{v}{\xi}\left(|D \xi|^{2}+\left(\mu u^{\delta}\right)^{2}\right)
\end{aligned}
$$

Moreover, using Young's inequality $a \cdot b \leq \frac{1}{2}\left(a^{2}+b^{2}\right)$ we obtain an estimate for the second term in (3.3)

$$
\begin{aligned}
v\left(\xi_{t}-\sum_{i, j} a_{i j}^{p, \delta}\left(D u^{\delta}\right) \xi_{i j}\right) & \leq v\left|\xi_{t}\right|+\frac{n}{p} v\left|D^{2} \xi\right|+\frac{|p-2|}{p} v\left|D^{2} \xi\right| \\
& \leq \eta \lambda v^{2}+\frac{1}{4 \lambda \eta}\left[\left|\xi_{t}\right|+\frac{n+|p-2|}{p}\left|D^{2} \xi\right|\right]^{2}
\end{aligned}
$$


with $a:=\sqrt{2 \eta \mu} \cdot v$ and $b:=\sqrt{\frac{1}{2 \eta \mu}}\left(\left|\xi_{t}\right|+\frac{n+|p-2|}{p}\left|D^{2} \xi\right|\right)$ for some $\eta>0$. With all these inequalities and using (3.5) we obtain for the fifth term in (3.3)

$$
\begin{aligned}
2 \lambda \sum_{i, j} a_{i j}^{p, \delta}\left(D u^{\delta}\right) u_{i}^{\delta} u_{j}^{\delta} & =2 \lambda\left|D u^{\delta}\right|^{2}\left(\frac{1}{p}+\frac{p-2}{p} \frac{\left|D u^{\delta}\right|^{2}}{\left|D u^{\delta}\right|^{2}+\delta}\right) \\
& \leq \frac{1+|p-2|}{p} \frac{5 v}{\xi}\left(|\nabla \xi|^{2}+\left(\lambda u^{\delta}\right)^{2}\right)+\eta \mu v^{2} \\
& +\frac{1}{4 \eta \mu}\left(\left|\xi_{t}\right|+\frac{n+p-2}{p}\left|D^{2} \xi\right|\right)^{2} \\
& +4 \frac{1+|p-2|}{p} \frac{v}{\xi}\left(|\nabla \xi|^{2}+\left(\mu u^{\delta}\right)^{2}\right)-2 \mu u^{\delta}\left(\zeta_{\varepsilon}\left(u^{\delta}\right)+f_{\varepsilon}\right) \\
& -\frac{\xi}{v} \sum_{k}\left\{D_{k} f_{\varepsilon} \cdot u_{k}^{\delta}+\varepsilon^{-2} \zeta^{\prime}\left(u_{k}^{\delta}\right)^{2}\right\} \\
& \leq 10 \frac{v}{\xi}\left(|\nabla \xi|^{2}+\left(\mu u^{\delta}\right)^{2}\right)+\eta \mu v^{2} \\
& +\frac{1}{4 \eta \mu}\left(\left|\xi_{t}\right|+\frac{n+|p-2|}{p}\left|D^{2} \xi\right|\right)-2 \mu u\left(\zeta_{\varepsilon}\left(u^{\delta}\right)+f_{\varepsilon}\right) \\
& -\frac{\xi}{v} \sum_{k}\left\{D_{k} f_{\varepsilon} \cdot u_{k}+\varepsilon^{-2} \zeta^{\prime} u_{k}^{2}\right\} .
\end{aligned}
$$

Now, using the Young's inequality with $a=10 \frac{1}{\sqrt{2 \eta \mu}} \frac{\left(|\nabla \xi|^{2}+\left(\lambda u^{\delta}\right)^{2}\right)}{\xi}$ and $b=\sqrt{2 \eta \mu} \cdot v$ and noting that, if $\left|\nabla u^{\delta}\left(x_{0}, t_{0}\right)\right| \geq 1$ and $0<\delta \leq 1 / 2$, we can bound from below the expression

$$
\left(\frac{8}{9} \frac{p-1}{p}\right) \mu v^{2} \leq 2 \mu\left|\nabla u^{\delta}\right|^{2}\left(\frac{1}{p}+\frac{p-2}{p} \frac{\left|\nabla u^{\delta}\right|^{2}}{\left|\nabla u^{\delta}\right|^{2}+\delta}\right) .
$$

Thus, we get that

$$
\begin{aligned}
\left(\frac{8}{9} \frac{p-1}{p}-2 \eta\right) \xi^{2} v^{2} & \leq \frac{25}{\eta \mu^{2}}\left(\left(|\nabla \xi|^{2}+\left(\mu u^{\delta}\right)^{2}\right)^{2}\right) \\
& +\frac{1}{4 \eta \mu^{2}} \xi^{2}\left(\left|\xi_{t}\right|+\frac{n+|p-2|}{p}\left|D^{2} \xi\right|\right)^{2}+2 \xi^{2} u^{\delta}\left(\zeta_{\varepsilon}\left(u^{\delta}\right)+f_{\varepsilon}\right) \\
& +\frac{\xi^{3}}{v} \mu^{-1} \sum_{k}\left\{D_{k} f_{\varepsilon} \cdot u_{k}^{\delta}+\varepsilon^{-2} \zeta^{\prime}\left(u_{k}^{\delta}\right)^{2}\right\} .
\end{aligned}
$$

The coefficient $\left(\frac{8}{9} \frac{p-1}{p}-2 \eta\right)$ is supposed to be positive because we divide by it and want to preserve the same direction of the inequality. Note that, if $p \rightarrow 1$ the coefficient becomes negative. But for fixed $p>1$, we can choose $\eta=\eta(p)>0$ such that

$$
\left(\frac{8}{9} \frac{p-1}{p}-2 \eta\right) \geq \frac{5}{9} \frac{p-1}{p}:=\frac{1}{c(p)} .
$$

Now, note that,

$$
2 \xi^{2} u^{\delta}\left(\zeta_{\varepsilon}\left(u^{\delta}\right)+f_{\varepsilon}\right) \geq 0
$$


and

$$
\begin{aligned}
\frac{\xi^{3}}{v} \mu^{-1} \sum_{k}\left\{D_{k} f_{\varepsilon} \cdot u_{k}^{\delta}+\varepsilon^{-2} \zeta^{\prime}\left(u_{k}^{\delta}\right)^{2}\right\} & =\frac{\xi^{2}}{v} \mu^{-1}\left(\sum_{k} D_{k} f_{\varepsilon} \cdot u_{k}^{\delta}+\varepsilon^{-2} \zeta^{\prime}\left|\nabla u^{\delta}\right|^{2}\right) \\
& \leq \frac{\xi^{2}}{v} \mu^{-1}\left(|| \nabla f_{\varepsilon} \| \cdot\left|\nabla u^{\delta}\right|+\varepsilon^{-2}\left|\zeta^{\prime}\right|\left|\nabla u^{\delta}\right|^{2}\right) \\
& \leq \frac{2}{\Gamma} \xi^{2}\left(\left\|\nabla f_{\varepsilon}\right\| \cdot \frac{\left|\nabla u^{\delta}\right|}{v}+2 \sup \left|\zeta^{\prime}\right| \frac{\left|\nabla u^{\delta}\right|^{2}}{v}\right) \\
& \leq \frac{2}{\Gamma} \xi^{2}\left(\left\|\nabla f_{\varepsilon}\right\|+2 \sup \left|\zeta^{\prime}\right|\left|\nabla u^{\delta}\right|^{2}\right) .
\end{aligned}
$$

Substituting (3.7) and (3.8) into (3.6), we obtain

$$
\begin{aligned}
\xi^{2} v^{2} & \leq \frac{c(p)}{\mu^{2}}\left[\left(|\nabla \xi|^{2}+\left(\mu u^{\delta}\right)^{2}\right)+\xi\left(\left|\xi_{t}\right|+\frac{n+|p-2|}{p}\left|D^{2} \xi\right|^{2}\right)\right]^{2} \\
& +\frac{2 \cdot c(p)}{\Gamma} \xi^{2}\left\|\nabla f_{\varepsilon}\right\|+\frac{2 \cdot c(p)}{\Gamma} \sup \left|\zeta^{\prime}\right| \xi^{2} v^{2}
\end{aligned}
$$

Thus,

$$
\begin{aligned}
\xi^{2}\left(1-\frac{2 \cdot c(p)}{\Gamma} \sup \left|\zeta^{\prime}\right|\right) v^{2} & \leq \frac{c(p)}{\mu^{2}}\left[\left(|\nabla \xi|^{2}+\left(\mu u^{\delta}\right)^{2}\right)+\xi\left(\left|\xi_{t}\right|+\frac{n+|p-2|}{p}\left|D^{2} \xi\right|^{2}\right)\right]^{2} \\
& +\quad+\frac{2 \cdot c(p)}{\Gamma}\left\|\nabla f_{\varepsilon}\right\| .
\end{aligned}
$$

Therefore, we can choose $\Gamma=\Gamma(p)>0$ such that

$$
1-\frac{2 \cdot c(p)}{\Gamma}\left\|\zeta^{\prime}\right\|_{\infty} \geq \frac{1}{2}
$$

This leads to the following inequality, at $\left(x_{0}, t_{0}\right)$

$$
\xi^{2} v^{2} \leq \frac{c(p)}{\mu^{2}}\left[\left(|\nabla \xi|^{2}+\left(\mu u^{\delta}\right)^{2}\right)+\xi\left(\left|\xi_{t}\right|+\frac{n+|p-2|}{p}\left|D^{2} \xi\right|^{2}\right)\right]^{2}+\kappa(p)
$$

where $\kappa(p):=\frac{2 \cdot c(p)}{\Gamma}\left\|\nabla f_{\varepsilon}\right\|_{\infty}$. Note that the constant $c(p)$ blows up for $p \rightarrow 1$. Now, fix $(x, t) \in \Omega_{T}$ and choose $\xi \in(0,1)$ such that $\xi\left(x_{0}, t_{0}\right)=1$ and

$$
\max \left\{\left\|D^{2} \xi\right\|_{\infty},\|\nabla \xi\|_{\infty},\left\|\xi_{t}\right\|_{\infty}\right\} \leq \frac{1}{\operatorname{dist}\left((x, t), \partial_{p} \Omega_{T}\right)} .
$$

Then

$$
\begin{aligned}
\left|\nabla u^{\delta}(x, t)\right| & \leq w(x, t) \leq w\left(x_{0}, t_{0}\right)=\xi\left(x_{0}, t_{0}\right) v\left(x_{0}, t_{0}\right)+\frac{\Gamma}{2 \varepsilon^{2}}\left(u^{\delta}\left(x_{0}, t_{0}\right)\right)^{2} \\
& \leq \frac{C(p, n)}{\mu}\left(\left\|D^{2} \xi\right\|_{\infty}+\Gamma+\|\nabla \xi\|_{\infty}^{2}+\left\|\xi_{t}\right\|_{\infty}\right)+\kappa(p) \\
& \leq C(n, p)\left(1+\frac{1}{\operatorname{dist}\left((x, t), \partial_{p} \Omega_{T}\right)}+\frac{1}{\operatorname{dist}\left((x, t), \partial_{p} \Omega_{T}\right)^{2}}\right) .
\end{aligned}
$$

We consider two cases. First we suppose that $\operatorname{dist}\left((x, t), \partial_{p} \Omega_{T}\right) \leq 1$. This implies

$$
\begin{aligned}
\left|\nabla u^{\delta}(x, t)\right| & \leq C(n, p)\left(1+\frac{2}{\operatorname{dist}\left((x, t), \partial_{p} \Omega_{T}\right)^{2}}\right) \\
& \leq C(n, p)\left(1+\frac{1}{\operatorname{dist}\left((x, t), \partial_{p} \Omega_{T}\right)^{2}}\right)
\end{aligned}
$$


For the sake of simplicity let $C(n, p)$ be a generic constant. We obtain, for $\operatorname{dist}\left((x, t), \partial_{p} \Omega_{T}\right) \geq$ 1 ,

$$
\begin{aligned}
\left|\nabla u^{\delta}(x, t)\right| & \leq C(n, p)\left(2+\frac{1}{\operatorname{dist}\left((x, t), \partial_{p} \Omega_{T}\right)^{2}}\right) \\
& \leq C(n, p)\left(1+\frac{1}{\operatorname{dist}\left((x, t), \partial_{p} \Omega_{T}\right)^{2}}\right)
\end{aligned}
$$

Finally we treat the case when the maximum point $\left(x_{0}, t_{0}\right)$ of $w$ is attained on the parabolic boundary $\partial_{p} \Omega_{T}$, then

$$
\begin{aligned}
\left|\nabla u^{\delta}(x, t)\right| & \leq v(x, t) \leq w(x, t) \leq w\left(x_{0}, t_{0}\right)=\mu\left(u^{\delta}\left(x_{0}, t_{0}\right)\right)^{2} \\
& \leq\|\varphi\|_{\infty} \leq \bar{C}\left(p, n,\|\varphi\|_{\infty}\right)\left(1+\frac{1}{\operatorname{dist}\left((x, t), \partial_{p} \Omega_{T}\right)^{2}}\right)
\end{aligned}
$$

because $\xi \equiv 0$ on $\partial_{p} \Omega_{T}$.

Using the Proposition 3.1, we obtain the following result

Corollary 3.1. If $\left\{u_{\varepsilon}\right\}_{\varepsilon>0}$ is a family of solutions of $\left(E_{\varepsilon}\right)$, and (2.3)-(2.4) hold, then there exists a constant $\bar{L}=L\left(\tau, \lambda, \Lambda, c_{0}, c_{1},\|\nabla f\|_{\infty},\|\varphi\|_{\infty}, K\right)>0$, independent of $\varepsilon \in(0,1)$, such that

$$
\left|\nabla u_{\varepsilon}(x, t)\right| \leq \bar{L}\left(1+\frac{1}{\operatorname{dist}\left((x, t), \partial_{p} \Omega_{T}\right)^{2}}\right), \forall(x, t) \in \Omega_{T} .
$$

Proof. Fix $\epsilon>0$. Initially, note that the approximating functions $u_{\varepsilon, \delta}$ converge locally uniformly to the viscosity solution $u_{\varepsilon}$ of the singular perturbation problem (see Section 3.2 in [11])

$$
\mathcal{L} u_{\varepsilon}-\partial_{t} u_{\varepsilon}=f_{\epsilon}+\zeta_{\epsilon}(u)
$$

as $\delta \rightarrow 0$. Since $u_{\varepsilon, \delta}$ is uniformly locally Lipschitz continuous, there exists for every $x \in \Omega$ a neighborhood $U_{x} \subset \Omega$ of $x$ and a constant $L>0$ such that

$$
\begin{aligned}
\left|u_{\varepsilon}(y, t)-u_{\varepsilon}(z, t)\right| & \leq\left|u_{\varepsilon}(y, t)-u_{\varepsilon, \delta}(y, t)\right|+\left|u_{\varepsilon, \delta}(y, t)-u_{\varepsilon, \delta}(z, t)\right| \\
& +\left|u_{\varepsilon, \delta}(z, t)-u_{\varepsilon}(z, t)\right| \leq 2 \tilde{\epsilon}(\delta)+L \cdot|y-z|
\end{aligned}
$$

for all $y, z \in U_{x}$ and fixed $t$, where $\tilde{\epsilon} \rightarrow 0$ for $\delta \rightarrow 0$ and $L$ independent of $\varepsilon$ and $\delta$. Then Rademacher's Theorem, e.g., stated in [10, provides that the gradient $\nabla u_{\varepsilon}(x, t)$ exists almost everywhere in $\Omega_{T}$. Let $(x, t) \in \Omega_{T}$ be a point where the gradient of $u$ exists and $B_{r}(x, t) \Subset \Omega_{T}$ be a ball of radius $r>0$ around $(x, t)$, then by Proposition 3.1

$$
\left|\nabla u_{\varepsilon, \delta}(y, s)\right|_{B_{r}(x, t)} \mid \leq \bar{C}\left(1+\frac{1}{\min _{(y, s) \in B_{r}(x, t)}\left(\operatorname{dist}\left((y, s), \partial_{p} \Omega_{T}\right)\right)^{2}}\right)
$$

for all $(y, s) \in B_{r}(x, t)$. Passing to the limit $\delta \rightarrow 0$;

$$
\left|\nabla u_{\varepsilon}(y, s)\right|_{B_{r}(x, t)} \mid \leq \bar{C}\left(1+\frac{1}{\min _{(y, s) \in B_{r}(x, t)}\left(\operatorname{dist}\left((y, s), \partial_{p} \Omega_{T}\right)\right)^{2}}\right)
$$

in points $(y, s)$ where the gradient exists since the right hand side is independent of $\delta$. Thus, due to the Lebesgue-Besicovitch Differentiation Theorem in [10, we have 
for almost every point $(x, t) \in \Omega_{T}$,

$$
\begin{aligned}
\left|\nabla u_{\varepsilon}(x, t)\right| & \leq \lim _{r \rightarrow 0} f_{B_{r}(x, t)}\left|\nabla u_{\varepsilon}(y, s)\right| d(y, s) \\
& \leq \bar{C} \lim _{r \rightarrow 0}\left(1+\frac{1}{\min _{(y, s) \in B_{r}(x, t)}\left(\operatorname{dist}\left((y, s), \partial_{p} \Omega_{T}\right)\right)^{2}}\right) \\
& =\bar{C}\left(1+\frac{1}{\operatorname{dist}\left((x, t), \partial_{p} \Omega_{T}\right)^{2}}\right) .
\end{aligned}
$$

As an immediate consequence we have the following result.

Corollary 3.2 (Local Lipschitz regularity). Let $\left\{u_{\varepsilon}\right\}_{\varepsilon>0}$ be a family of solutions of (E) . Let $K \subset \Omega_{T}$ be a compact set and $\tau>0$ be such that $\mathcal{N}_{\tau}^{-}(K) \subset \Omega_{T}$. If (2.3)(2.4) hold, then there exists a constant $L=L\left(\tau, \lambda, \Lambda, c_{0}, c_{1},\|\nabla f\|_{\infty},\|\varphi\|_{\infty}, K\right)>0$ such that

$$
\left|\nabla u_{\varepsilon}(x, t)\right| \leq L, \quad \forall(x, t) \in K
$$

Proof. For $\left(x_{0}, t_{0}\right) \in K$, consider the function

$$
w_{\varepsilon, r}(x, t):=\frac{1}{r} u_{\varepsilon}\left(x_{0}+r x, t_{0}+r^{2} t\right) .
$$

For $r \in(0, \tau)$ we have that $w_{\varepsilon, r}$ is a solution of

$$
\begin{aligned}
\mathcal{L} w_{\varepsilon, r}-\partial_{t} w_{\varepsilon, r} & =\zeta_{\varepsilon / r}\left(w_{\varepsilon, r}\right)+r f_{\varepsilon} \\
: & =g_{\varepsilon}(x, t)
\end{aligned}
$$

in $B_{1} \times(-1,0)$. The result now follows from Corollary 3.1 .

3.2. Uniform regularity in time. Next, as was mentioned above, using the uniform Lipschitz continuity in the space variables, we obtain the uniform Hölder continuity in time. First, we need the following lemma.

Lemma 3.1. Let $0 \leq u \in C\left(\bar{B}_{1}(0) \times\left[0,1 /\left(4 n+M_{0}\right)\right]\right)$ be such that

$$
\left|\mathcal{L} u-\partial_{t} u\right| \leq M_{0} \quad \text { in } \quad\{u>1\},
$$

for some $M_{0}>0$, and $|\nabla u| \leq L$, for some $L>0$. Then there exists a constant $C=C(L)$ such that

$$
|u(0, t)-u(0,0)| \leq C, \quad \text { if } \quad 0 \leq t \leq \frac{1}{4 n+M_{0}} .
$$

Proof. This lemma is a slight modification of [16, Lemma 4.1]. Without loss of generality we may assume that $L>1$. We denote

$$
c(p):=\frac{n p \Lambda}{n+p-2}
$$

where $\Lambda:=\max \left\{\frac{1}{p}, \frac{p-1}{p}\right\}$ denotes the greatest eigenvalue. We divide the proof into two steps.

Step 1. First we claim that, if

$$
Q_{t_{0}, t_{1}}:=B_{1}(0) \times\left(t_{0}, t_{1}\right) \subset\{u>1\} \quad \text { for } \quad t_{1}-t_{0} \leq \frac{1}{4 n+M_{0}},
$$

then

$$
\left|u\left(0, t_{1}\right)-u\left(0, t_{0}\right)\right| \leq 2 L
$$


In fact, let

$$
h^{ \pm}(x, t):=u\left(0, t_{0}\right) \pm L \pm \frac{2 L}{\Lambda} c(p)|x|^{2} \pm\left(4 n L+M_{0}\right)\left(t-t_{0}\right) .
$$

Thus, Then for the specific $h^{ \pm}$we obtain

$$
\begin{aligned}
\partial_{t} h^{+}-\mathcal{L} h^{+} & =\left(4 n L+M_{0}\right)-\left(\frac{n}{p}+\frac{p-2}{p}\right) \frac{4 L}{\Lambda} c(p) \\
& =M_{0} \\
\partial_{t} h^{-}-\mathcal{L} h^{-} & =-\left(4 n L+M_{0}\right)+\left(\frac{n}{p}+\frac{p-2}{p}\right) \frac{4 L}{\Lambda} c(p) \\
& =-M_{0}
\end{aligned}
$$

Set

$$
t_{2}:=\sup _{t_{0} \leq \bar{t} \leq t_{1}}\left\{\bar{t}:\left|u(0, t)-u\left(0, t_{0}\right)\right| \leq 2 L, \forall t_{0} \leq t \leq \bar{t}\right\} .
$$

So $t_{0}<t_{2} \leq t_{1}$ is such that

$$
\left|u(0, t)-u\left(0, t_{0}\right)\right| \leq 2 L, \quad \text { for } \quad t \in\left[t_{0}, t_{2}\right) .
$$

Moreover, from the Lipschitz continuity in space, one has

$$
h^{-} \leq u \leq h^{+} \quad \text { on } \quad \partial_{p} Q_{t_{0}, t_{2}} .
$$

On the other hand,

$$
\begin{aligned}
\partial_{t} h^{-}-\mathcal{L} h^{-} & \leq-M_{0} \leq \partial_{t} u-\Delta_{p}^{N} u \\
& \leq M_{0} \leq \partial_{t} h^{+}-\mathcal{L} h^{+} .
\end{aligned}
$$

Therefore, by comparison principle (see Proposition 2.1)

$$
h^{-} \leq u \leq h^{+} \quad \text { in } \quad Q_{t_{0}, t_{2}} .
$$

In particular, since $t_{2}-t_{0} \leq t_{1}-t_{0} \leq \frac{1}{4 n+M_{0}}$ and $L>1$ one has

$$
\left|u\left(0, t_{2}\right)-u\left(0, t_{0}\right)\right|<2 L .
$$

Because of the strict inequality above, we may take $t_{2}=t_{1}$ and therefore the claim is proved.

Step 2. Let us consider now the cylinder $Q_{0, t}$ with $0<t \leq \frac{1}{4 n+M_{0}}$.

If $Q_{0, t} \subset\{u>1\}$, we apply Step 1 to get

$$
|u(0, t)-u(0,0)| \leq 2 L .
$$

If $Q_{0, t} \nsubseteq\{u>1\}$, let $0 \leq t_{1} \leq t_{2} \leq t$ and $x_{1}, x_{2} \in \bar{B}_{1}(0)$ be such that

$$
0 \leq u\left(x_{1}, t_{1}\right) \leq 1, \quad 0 \leq u\left(x_{2}, t_{2}\right) \leq 1
$$

and

$$
\left(\bar{B}_{1}(0) \times\left(0, t_{1}\right)\right) \cup\left(\bar{B}_{1}(0) \times\left(t_{2}, t\right)\right) \subset\{u>1\} .
$$

Then, Step 1 and the Lipschitz continuity in space provide

$$
\begin{aligned}
|u(0, t)-u(0,0)| & \leq\left|u(0, t)-u\left(0, t_{2}\right)\right|+\left|u\left(0, t_{2}\right)-u\left(x_{2}, t_{2}\right)\right|+\left|u\left(x_{2}, t_{2}\right)\right| \\
& +\left|u\left(x_{1}, t_{1}\right)\right|+\left|u\left(x_{1}, t_{1}\right)-u\left(0, t_{1}\right)\right|+\left|u\left(0, t_{1}\right)-u(0,0)\right| \\
& \leq 2(2 L+L+1),
\end{aligned}
$$

which completes the proof.

We are now ready to prove uniform Hölder continuity of solutions in time. 
Proposition 3.2. Let $\left\{u_{\varepsilon}\right\}_{\varepsilon>0}$ be a family of solutions of $E_{\varepsilon}$. Let $K \subset \Omega_{T}$ be compact and $\tau>0$ be such that $\mathcal{N}_{2 \tau}(K) \subset \Omega_{T}$. If (2.3)-(2.4) hold, then there exists a constant $C>0$, independent of $\varepsilon$, such that

$$
\left|u_{\varepsilon}(x, t+\Delta t)-u_{\varepsilon}(x, t)\right| \leq C|\Delta t|^{1 / 2}, \quad \text { for }(x, t),(x, t+\Delta t) \in K .
$$

Proof. Let $r \in(0, \tau), \varepsilon \leq r,\left(x_{0}, t_{0}\right) \in K$ and $w_{\varepsilon, r}(x, t), g_{\varepsilon}(x, t)$ be as in the proof of Corollary 3.2. From (2.3) and (2.4) we get, in the set $\left\{w_{\varepsilon, r} \geq \varepsilon / r\right\}$,

$$
0 \leq g_{\varepsilon}(x, t) \leq r f_{\varepsilon}(x)+\frac{r}{\varepsilon} \zeta\left(\frac{r}{\varepsilon} w_{\varepsilon, r}\right) \leq \tau c_{1}=: C_{\star} .
$$

Also $\left|\nabla w_{\varepsilon, r}(x, t)\right| \leq L$. Therefore, we may apply Lemma 3.1, with $M_{0}=C_{\star}$, to obtain

$$
\left|w_{\varepsilon, r}(0, t)-w_{\varepsilon, r}(0,0)\right| \leq C, \quad \text { for } \quad 0 \leq t \leq \frac{1}{4 n+C_{\star}},
$$

or in other terms

$$
\left|u_{\varepsilon}\left(x_{0}, t_{0}+r^{2} t\right)-u_{\varepsilon}\left(x_{0}, t_{0}\right)\right| \leq C r, \quad \text { for } 0 \leq t \leq \frac{1}{4 n+C_{\star}} .
$$

In particular, for $r \in(0, \tau)$, one has

$$
\left|u_{\varepsilon}\left(x_{0}, t_{0}+\frac{r^{2}}{4 n+C_{\star}}\right)-u_{\varepsilon}\left(x_{0}, t_{0}\right)\right| \leq C r .
$$

Now if $\left(x_{0}, t_{0}+\Delta t\right) \in K$ and $0<\Delta t<\frac{\tau^{2}}{4 n+C_{\star}}$, taking $r=\Delta t^{1 / 2} \sqrt{4 n+C_{\star}}$ in (3.9) leads to

$$
\left|u_{\varepsilon}\left(x_{0}, t_{0}+\Delta t\right)-u_{\varepsilon}\left(x_{0}, t_{0}\right)\right| \leq C \sqrt{4 n+C_{\star}} \Delta t^{1 / 2} .
$$

On the other hand, if $\Delta t \geq \frac{\tau^{2}}{4 n+C_{\star}}$, thus we get

$$
\left|u_{\varepsilon}\left(x_{0}, t_{0}+\Delta t\right)-u_{\varepsilon}\left(x_{0}, t_{0}\right)\right| \leq 2 \Upsilon \leq \frac{2 \Upsilon}{\tau} \sqrt{4 n+C_{\star}} \Delta t^{1 / 2},
$$

which completes the proof.

We are interested in geometric proprieties of the limiting function

$$
u:=\lim _{k \rightarrow \infty} u_{\varepsilon_{k}},
$$

for a subsequence $\varepsilon_{k} \rightarrow 0$. From Theorem 2.2 the family $\left\{u_{\varepsilon}\right\}$ is pre-compact in $\operatorname{Lip}(1,1 / 2)\left(\Omega_{T}\right)$. Hence, up to a subsequence, there exists a limiting function $u$, obtained as the uniform limit of $u_{\varepsilon}$, as $\varepsilon \rightarrow 0$. One readily verifies that the limiting function $u$ satisfies (see Theorem 5.1 in [16] )

(1) $u$ is a solution of

$$
\mathcal{L} u-u_{t}=f \cdot \chi_{\{u>0\}},
$$

where $f$ is the uniform limit of $f_{\varepsilon}$, with $f$ satisfing (2.3)-(2.4);

(2) the function $t \mapsto u(x, t)$ is non-decreasing in time. 


\section{SCALing BARRIERS AND GEOMETRIC NONDEGENERACY}

In this section we establish the exact growth of the solution near the free boundary, from which we deduce the porosity of its time level sets (see Theorem 2.4). The proof is quite similar to those in [16, but for the reader's convenience we decided to give the details.

Lemma 4.1. Let $u \in C\left(Q_{1}\right)$ be a viscosity solution to

$$
\mathcal{L} u-u_{t}=f \quad \text { in } \quad U^{+}:=\{u>0\} .
$$

Then for every $(z, s) \in \overline{\{u>0\}}$ and $r>0$ with $Q_{r}(z, s) \subset Q_{1}$ we have

$$
\sup _{(x, t) \in \partial_{p} Q_{r}^{-}(z, s)} u(x, t) \geq \mu_{0} r^{2}+u(z, s),
$$

where $\mu_{0}=\min \left(\frac{p c_{0}}{4(n+p-2)}, \frac{c_{0}}{2}\right)$.

Proof. Suppose that $(z, s) \in\{u>0\}$, and, for small $\delta>0$, set

$$
\begin{aligned}
\omega^{\delta}(x, t) & :=u(x, t)-(1-\delta) u(z, s) \\
\psi(x, t) & :=\left(\frac{p c_{0}}{4(n+p-2)}\right)|x-z|^{2}-\left(\frac{c_{0}}{2}\right)(t-s) .
\end{aligned}
$$

Since $D_{i j} \psi=\frac{p c_{0}}{2(n+p-2)} \delta_{i j}$ then

$$
\begin{aligned}
\mathcal{L} \psi-\partial_{t} \psi & =\frac{1}{p} \Delta \psi+\frac{p-2}{p}\left\langle D^{2} \psi \cdot \frac{\nabla \psi}{|\nabla \psi|}, \frac{\nabla \psi}{|\nabla \psi|}\right\rangle \\
& =\frac{1}{p} \cdot \frac{n p c_{0}}{2(n+p-2)}+\frac{p-2}{p} \cdot \frac{p c_{0}}{2(n+p-2)}+\frac{c_{0}}{2} \\
& =\frac{n c_{0}}{2(n+p-2)}+\frac{(p-2) c_{0}}{2(n+p-2)}+\frac{c_{0}}{2} \\
& =\frac{c_{0}}{2}+\frac{c_{0}}{2}=c_{0} \\
& \leq f(x, t)=\Delta_{p}^{N} u-\partial_{t} u \\
& =\mathcal{L} \omega^{\delta}-\partial_{t} \omega^{\delta} .
\end{aligned}
$$

Moreover, $\omega^{\delta} \leq \psi$ on $\partial\{u>0\} \cap Q_{r}^{-}(z, s)$. Note that we can not have

$$
\omega^{\delta} \leq \psi \quad \text { on } \quad \partial_{p} Q_{r}^{-}(z, s) \cap\{u>0\},
$$

because otherwise we could apply the comparison principle(see Proposition 2.1) to obtain

$$
\omega^{\delta} \leq \psi \quad \text { in } \quad Q_{r}^{-}(z, s) \cap\{u>0\},
$$

which contradicts the fact that $\omega^{\delta}(z, s)=\delta u(z, s)>0=\psi(z, s)$. Hence, for $(y, \tau) \in \partial_{p} Q_{r}^{-}(z, s)$ we must have

$$
\omega^{\delta}(y, \tau)>\psi(y, \tau)=\mu_{0} r^{2} .
$$

Letting $\delta \rightarrow 0$ in the last inequality we conclude the proof. 
4.1. A class of functions in the unit cylinder. Next, we establish the growth rate of the solution near the free boundary, which is known for $p$-parabolic variational problems (see [18]) but is new in the fully nonlinear framework. We start by introducing a class of functions.

Definition 4.1. We say that a function $u \in C\left(Q_{1}\right)$ is in the class $\Theta$ if $0 \leq u \leq 1$ in $Q_{1}, 0 \leq \mathcal{L} u-\partial_{t} u \leq c_{1}$ in $Q_{1}$ and $\partial_{t} u \geq 0$, in the viscosity sense, with $u(0,0)=0$.

The following Theorem gives the growth of the elements of the family $\Theta$. This completes a result proved in [16] for the fully nonlinear parabolic equations case.

Theorem 4.1. If $u \in \Theta$, then there is a constant $C_{0}=C_{0}\left(n, p, L, c_{1}\right)>0$ such that

$$
|u(x, t)| \leq C_{0}(d(x, t))^{2}, \quad \forall(x, t) \in Q_{1 / 2},
$$

where

$$
d(x, t):= \begin{cases}\sup \left\{r: Q_{r}(x, t) \subset\{u>0\}\right\}, & \text { if }(x, t) \in\{u>0\} \\ 0, & \text { otherwise. }\end{cases}
$$

To prove Lemma 4.1, we need to introduce some notation. Set

$$
S(r, u, z, s):=\sup _{Q_{r}^{-}(z, s)} u \text {. }
$$

For $u \in \Theta$, we define

$$
H(u, z, s):=\left\{j \in \mathbb{N} \cup\{0\}: S\left(2^{-j}, u, z, s\right) \leq M S\left(2^{-j-1}, u, z, s\right)\right\},
$$

where $M:=4 \max \left(1, \frac{1}{\mu_{0}}\right)$, with $\mu_{0}$ as in Lemma 4.1. When $(z, s)$ is the origin, we suppress the point dependence. As in [16], we first show a weaker version of the inequality.

Lemma 4.2. If $u \in \Theta$, then there is a constant $C_{1}=C_{1}\left(n, c_{1}\right)>0$ such that

$$
S\left(2^{-j-1}, u\right) \leq C_{1} 2^{-2 j}, \quad \forall j \in H(u) .
$$

Proof. First, note that $H(u) \neq \emptyset$ because $0 \in H(u)$. Indeed, using Lemma 4.1, we have

$$
S(1, u) \leq 1=4\left(\frac{1}{\mu_{0}}\right) \mu_{0} 2^{-2} \leq 4\left(\frac{1}{\mu_{0}}\right) S\left(2^{-1}, u\right) \leq M S\left(2^{-1}, u\right) .
$$

Next, suppose the conclusion of the lemma fails. Then, for every $k \in \mathbb{N}$, there is $u_{k} \in \Theta$ and $j_{k} \in H\left(u_{k}\right)$ such that

$$
S\left(2^{-j_{k}-1}, u_{k}\right) \geq k 2^{-2 j_{k}} .
$$

Define $v_{k}: Q_{1} \rightarrow \mathbb{R}$ by

$$
v_{k}(x, t):=\frac{u_{k}\left(2^{-j_{k}} x, 2^{-2 j_{k}} t\right)}{S\left(2^{-j_{k}-1}, u_{k}\right)}
$$

One easily verifies that

$$
\begin{gathered}
0 \leq v_{k} \leq 1 \quad \text { in } Q_{1}^{-} ; \quad 0 \leq \mathcal{L} v_{k}-\partial_{t} v_{k} \leq \frac{c_{1}}{k} \\
\sup _{Q_{1 / 2}^{-}} v_{k}=1 ; \quad v_{k}(0,0)=0 ; \quad \partial_{t} v_{k} \geq 0 \quad \text { in } Q_{1}^{-} .
\end{gathered}
$$


Therefore, from [2, Theorem 1.1] we deduce that $v_{k} \in C_{\mathrm{loc}}^{1+\alpha, \frac{1+\alpha}{2}}\left(Q_{1}^{-}\right)$is uniformly bounded, independently of $k$, for a constant $\alpha \in(0,1)$. It follows then from ArzeláAscoli theorem that there exists a subsequence, still denoted $v_{k}$, and a function $v \in C^{1+\alpha, \frac{1+\alpha}{2}}\left(\overline{Q_{3 / 4}^{-}}\right)$such that $v_{k} \rightarrow v$ and $\nabla v_{k} \rightarrow \nabla v$ uniformly in $\overline{Q_{3 / 4}^{-}}$. Moreover,

$$
\sup _{Q_{1 / 2}^{-}} v=1
$$

and using compactness arguments (see [17, Theorem 2.10])

$$
\partial_{t} v-\mathcal{L} v=0 \quad \text { in } \quad Q_{3 / 4}^{-}, \quad v(0,0)=0, \quad v \geq 0, \quad \partial_{t} v \geq 0
$$

in $Q_{3 / 4}^{-}$. Therefore, by the maximum principle (see [1]), we obtain $v \equiv 0$. This gives us a contradiction to [4.1], if we choose $k \gg 1$.

Proof of Theorem 4.1. Using Lemma 4.2, it follows exactly as in [16, Lemma 6.3] that

$$
S\left(2^{-j}, u\right) \leq 4 C_{1} 2^{-2 j}, \forall j \in \mathbb{N} .
$$

Now, fix $r \in(0,1)$, by choosing $j \in \mathbb{N}$ such that $2^{-j-1} \leq r \leq 2^{-j}$, one has

$$
\sup _{Q_{r}^{-}(0,0)} u \leq \sup _{Q_{2^{-j}}^{-}} u \leq 4 C_{1} 2^{-2 j}=16 C_{1} 2^{-2 j-2} \leq 16 C_{1} r^{2},
$$

i.e.,

$$
S(r, u) \leq 16 C_{1} r^{2} .
$$

To obtain a similar estimate for $u$ over the whole cylinder (and not only over its lower half) we use a barrier from above. Set

$$
\omega(x, t):=A_{1}|x|^{2}+A_{2} t
$$

where $A_{2}=\frac{2(n+p-2)}{p} A_{1}$ and $A_{1}>0$. Then in $Q_{1}^{+}=B_{1}(0) \times(0,1)$ one gets that

$$
\begin{aligned}
\mathcal{L} \omega-\partial_{t} \omega & =\frac{2 A_{1} n}{p}+2 A_{1} \frac{p-2}{p}-A_{2} \\
& =2\left(\frac{n}{p}+\frac{p-2}{p}\right) A_{1}-A_{2}=0 \\
& \leq \mathcal{L} u-\partial_{t} u .
\end{aligned}
$$

If $A_{1}$ is large enough, then $\omega \geq u$ on $\partial_{p} Q_{1}^{+}$, where for the estimate on $\{t=0\}$ we used $S(r, u) \leq 16 C_{1} r^{2}$ from (4.3). Hence, by the comparison principle (see [17, Theorem 2.9] ) one has $\omega \geq u$ in $Q_{1}^{+}$. Therefore

$$
\sup _{Q_{r}(0,0)} u \leq C_{0} r^{2}
$$

for a constant $C_{0}>0$.

Proof of Theorem 2.4. Using Theorem 4.1 the proof follows exactly as the one of [16. 


\section{REFERENCES}

[1] A. Argiolas, F. Charro, I. Peral, On the Aleksandrov-Bakel'man-Pucci estimate for some Elliptic and Parabolic Nonlinear Operators. Archive for Rational Mechanics and Analysis; 202(3) (2011); 875-917.

[2] A. Attouchi, M. Parviainem, Hölder regularity for the gradient of the inhomogeneous parabolic normalized p-Laplacian. Communications in Contemporary Mathematics 20(4) (2017)

[3] A. Banerjee, N. Garofalo, Gradient bounds and monotonicity of the energy for some nonlinear singular diffusion equations, Indiana Univ. Math. J. 62 (2) (2013) 699?736.

[4] L. Caffarelli, Uniform Lipschitz regularity of a singular perturbation problem, Differ. Integral Equ. 8 (1995), 1585-1590.

[5] L. Caffarelli, C. Lederman, N. Wolanski, Uniform estimates and limits for a two phase parabolic singular perturbation problem, Indiana Univ. Math. J. 46 (1997), 453-489.

[6] L. Caffarelli ,C. Lederman, N. Wolanski, Pointwise and viscosity solutions for the limit of a two phase parabolic singular perturbation problem, Indiana Univ. Math. J. 46 (1997), 719-740.

[7] L. Caffarelli, J.L. Vázquez, A free boundary problem for the heat equation arising in flame propagation, Trans. Amer. Math. Soc. 347 (1995), 411-441.

[8] F. Charro, G. De Phillippis, A. Di Castro, A. Máximo, On the Aleksandrov-Bakelman-Pucci estimate for the infinity Laplacian. Calculus of Variations. November 2013, Volume 48, Issue $3 Đ 4$, pp $667 Đ 693$.

[9] M.G. Crandall, H. Ishii, and P.L. Lions, User's guide to viscosity solutions of second order partial differential equations, Bull. Amer. Math. Soc. 27 (1992), 1-67.

[10] L.C. Evans, R.F. Gariepy, Measure Theory and Fine Properties of Functions,CRC Press, Boca Raton, Ann Arbor and London, 1992.

[11] D. Kerstin , An evolution equation involving the normalized p-Laplacian. Communications on pure and applied analysis. Vol. 10, Number 1, January 2011.

[12] O.A. Ladyzenskaja, V.A. Solonnikov, N. Uralceva, Linear and quasilinear equations of parabolic type. Translated from the Russian by S. Smith. Translations of Mathematical Monographs, Vol. 23. American Mathematical Society, Providence, R.I., 1968.

[13] M. Montenegro, E. Teixeira, Gradient estimates for viscosity solutions of singular fully nonlinear elliptic equations, J. Funct. Anal. 259 (2010), 428-452.

[14] Y. Peres, O. Schramm, S. Sheffield, D. Wilson, Tug-of-war and the infinity Laplacian. J. Amer. Math. Soc., 22(1):167-210, 2009.

[15] G.C. Ricarte, E. Teixeira, Fully nonlinear singularly perturbed equations and asymptotic free boundaries, J. Funct. Anal. 261 (2011), 1624-1673.

[16] G.C. Ricarte, R. Teymurazyan,J.M. Urbano, Singularity perturbed fully nonlinear parabolic problems and their asymptotic free boundaries. https://arxiv.org/pdf/1604.01294.pdf

[17] L. Silvestre, J. Tianling, Hölder gradient estimates for parabolic homogeneous p-Laplacian equations. Journal de Mathématiques Pures et Appliquées. Volume 108, Issue 1, July 2017, Pages $63 Đ 87$.

[18] H. Shahgholian, Analysis of the free boundary for the p-parabolic variational problem ( $p \geq 2)$, Rev. Mat. Iberoamericana 19 (2003), 797-812.

[19] L. Wang, On the regularity theory of fully nonlinear parabolic equations: I, Comm. Pure Appl. Math. 45 (1992), 27-76.

[20] L. Wang, On the regularity theory of fully nonlinear parabolic equations: II, Comm. Pure Appl. Math. 45 (1992), 141-178.

[21] L. Wang, On the regularity theory of fully nonlinear parabolic equations: III, Comm. Pure Appl. Math. 45 (1992), 255-262.

Universidade Federal do Ceará - UfC, Department of Mathematics, Fortaleza - CE, BRAZIL - 60455-760.

E-mail address: ricarte@mat.ufc.br 